\title{
Platelets: a potential role in chronic respiratory diseases?
}

\author{
Mohamad Chebbo ${ }^{1}$, Catherine Duez $\mathbb{( D )}^{1}$, Marie C. Alessi ${ }^{1,2}$, Pascal Chanez ${ }^{1,3}$ and Delphine Gras $\mathbb{(}^{1}$ \\ ${ }^{1}$ Aix-Marseille Univ, INSERM, INRAE, Marseille, France. ${ }^{2} \mathrm{APHM}, \mathrm{CHU}$ de la Timone, Laboratoire d'hématologie, Marseille, France. ${ }^{3} \mathrm{APHM}$, \\ Hôpital NORD, Clinique des Bronches, Allergie et Sommeil, Marseille, France.
}

Corresponding author: Delphine Gras (delphine.gras@univ-amu.fr)

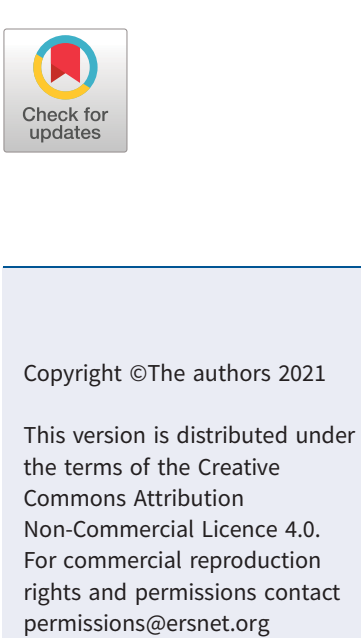

Received: 8 March 2021

Accepted: 5 June 2021

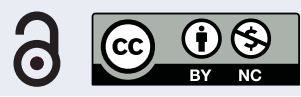

Shareable abstract (@ERSpublications)

In addition to their essential role in haemostasis and thrombosis, platelets are strong modulators of different immune responses, and could be involved in the physiopathology of several chronic airway diseases https://bit.ly/3cB6Xnj

Cite this article as: Chebbo M, Duez C, Alessi MC, et al. Platelets: a potential role in chronic respiratory diseases?. Eur Respir Rev 2021; 30: 210062 [DOI: 10.1183/16000617.0062-2021].

\section{Abstract}

Platelets are small anucleate cells known for their role in haemostasis and thrombosis. In recent years, an increasing number of observations have suggested that platelets are also immune cells and key modulators of immunity. They express different receptors and molecules that allow them to respond to pathogens, and to interact with other immune cells. Platelets were linked to the pathogenesis of some inflammatory disorders including respiratory diseases such as asthma and idiopathic pulmonary fibrosis. Here, we discuss the involvement of platelets in different immune responses, and we focus on their potential role in various chronic lung diseases.

\section{Introduction}

Platelets (also named thrombocytes) are small anucleate cells (2-4 $\mu \mathrm{m}$ in diameter) originating from the fragmentation of a large polyploid cell called "megakaryocyte". In the bone marrow, immature megakaryocytes undergo endomitosis in order to develop polyploid nuclei ( $2 \mathrm{n}$ to 128n). Polyploid megakaryocytes spread long cytoplasmic extensions known as pro-platelets and release them into the sinusoidal blood vessels, where they undergo further divisions and generate mature platelets. It has been estimated that one megakaryocyte can produce 100-1000 platelets [1-4]. The lifespan of platelets is $7-10$ days in humans and they are produced permanently to keep an average of $2-3 \times 10^{8}$ platelets per $\mathrm{mL}$ of blood [1, 2, 5-7].

Since their discovery, platelets have been known for their key role in haemostasis and thrombosis (figure 1), and researchers have focused on the involvement of these cells in blood disorders and cardiovascular diseases. However, it has been demonstrated that platelets are also able to modulate the immune response, through their interaction with immune cells in different pathological and physiological processes such as inflammation, tissue remodelling and infections.

In the context of respiratory diseases, many observations suggest a potential role of platelets in the physiopathology of these diseases, especially in asthma and stable COPD. Interestingly, a study using intravital microscopy in mice detected megakaryocytes producing platelets in pulmonary vasculature and estimated that the lung is responsible for $\sim 50 \%$ of total platelet production. In addition, the authors detected extravascular lung-resident megakaryocytes, not involved in platelet production. RNA sequencing analysis revealed that lung megakaryocytes possess an innate immunity phenotype compared to bone marrow megakaryocytes and could be involved in the regulation of the pulmonary immune response [10]. These findings were confirmed in a recent study showing that lung megakaryocytes had gene expression patterns similar to antigen-presenting cells and are able to process antigenic proteins and to induce CD4 ${ }^{+}$ T-cell activation in a major histocompatibility complex (MHC) class II dependent manner [8]. These observations are of great interest as they might unveil a new player in pulmonary immune homeostasis and diseases. 


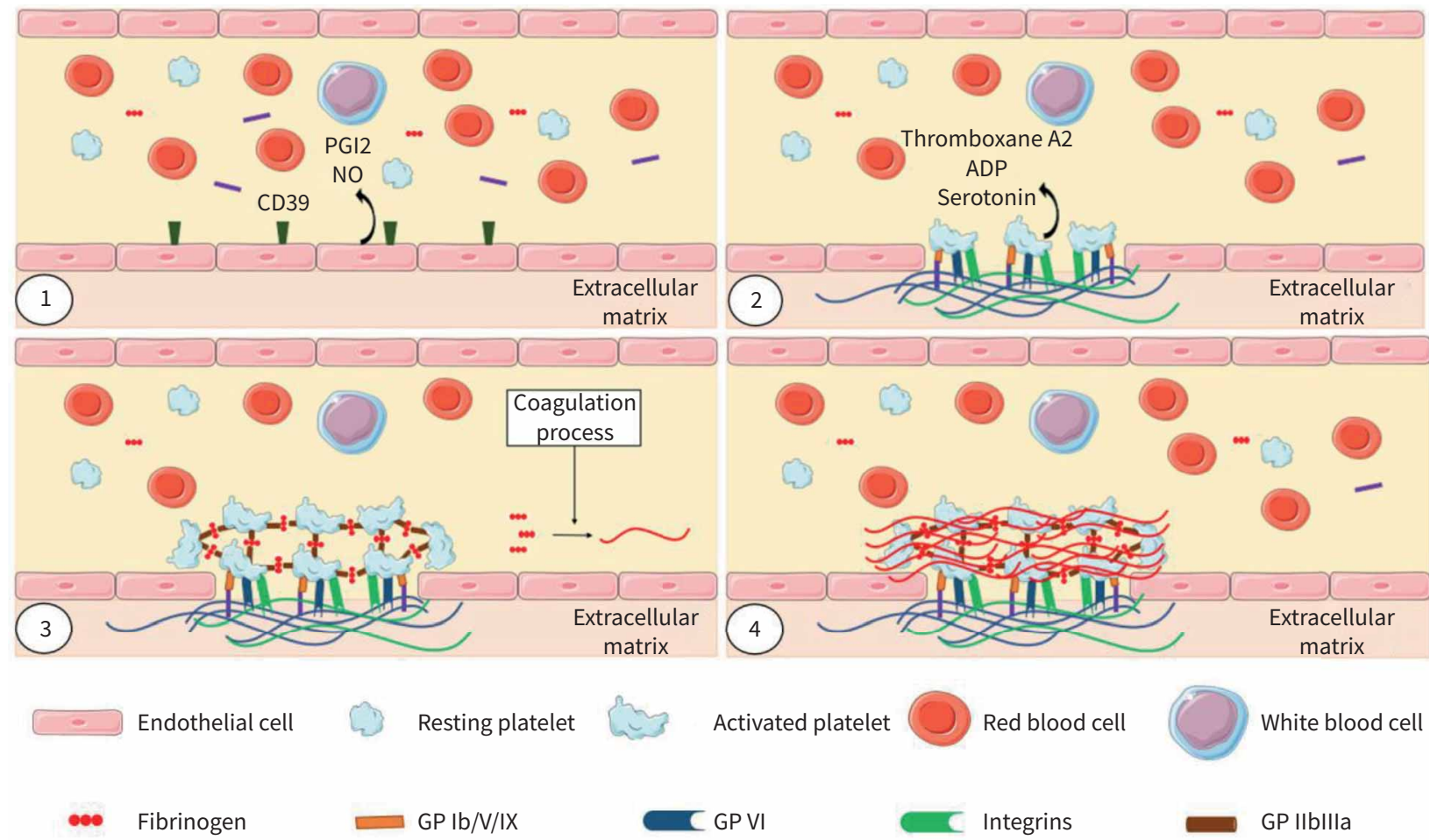

Von Willebrand factor
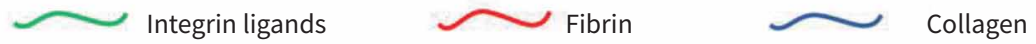

FIGURE 1 Platelets and haemostasis at high-shear rates. 1) In intact blood vessels, endothelial cells produce molecules that maintain platelets in an inactive state. 2) Following blood vessel damage, the blood components come in contact with the extracellular matrix, and plasmatic von Willebrand factor (VWF) anchors onto extracellular matrix collagen. Anchored vWF, by binding platelet glycoprotein (GP) complex Ib/V/IX, initiates platelet adhesion to injured vessel wall. This first binding sets the stage for a subsequent stable adhesion, mediated by the interaction between platelet GP VI and collagen, and between platelet integrins $(\alpha 2 \beta 1, \alpha 2 b \beta 3$ and $\alpha 6 \beta 1)$ and their ligands (collagen, fibrinogen and laminin, respectively). The stable adhesion triggers initial platelet activation and the release of soluble agonists such as ADP, serotonin and thromboxane A2. These agonists possess both an autocrine effect, which amplifies platelet activation, and a paracrine effect, which results in nearby platelet activation and recruitment to the site of injury. 3) and 4) The interaction of soluble agonists with their receptors and integrins with their ligands stimulates the increase of platelet cytosolic calcium concentrations, shape change and an "inside-out" signalling that activates the surface GPIIbIlla $(\alpha 2 b \beta 3)$ receptor, thus increases its binding affinity. Activated GPIIbllla mainly binds fibrinogen and fibrin, which leads to adjacent activated platelets cross-links and an "outside-in" signalling that stimulates essential platelet processes such as spreading, thrombus consolidation and clot retraction (negative feedback regulation) [8, 9]. PGI2: prostacyclin; NO: nitric oxide.

In this review, we focus on platelet immune functions and their impact on immune cell activation and differentiation in different stages of the immune response. In addition, we discuss the involvement of platelet in infectious and chronic respiratory diseases.

Platelets: small cells expressing a wide variety of molecules

Despite their small size, platelets contain several organelles such as mitochondria and three types of granules, i.e. dense granules, lysosomes and $\alpha$-granules, each containing proteins pre-synthetised in megakaryocytes and blood proteins internalised by endocytosis. Platelets are also able to synthesise new proteins from stored mRNA $[1,9,11]$.

Dense granules also known as $\delta$-granules (three to eight per platelet) encapsulate high concentrations of nucleotides (e.g. ADP and ATP), ionic calcium, polyphosphates and monoamines (e.g. serotonin and histamine) which potentiate platelet activation following their release. Lysosomes contain hydrolytic enzymes, essentially proteases, some of which are involved in the degradation of internalised receptors $[6,7,12]$. Finally, $\alpha$-granules are the most abundant granules in platelets (40-80 per platelet). They comprise many components which play critical roles in different biological activities, such as proteins involved in primary and secondary haemostasis (e.g. von Willebrand factor, fibrinogen), coagulation (e.g. 
factors V and XI, prothrombin), anticoagulation (e.g. antithrombin) and fibrinolysis (e.g. plasminogen), but also different families of adhesion proteins, such as integrins (e.g. $\alpha \mathrm{IIb}, \alpha 6, \beta 1, \beta 3$ ), selectins (e.g. P-selectin), immunoglobulin family receptors (e.g. glycoprotein (GP)VI, intercellular adhesion molecule (ICAM)-2) and leucine-rich repeat family receptors (e.g. GPIb-IX-V complex). In addition, they contain regulators of growth and angiogenesis (e.g. epidermal growth factor, angiopoietin-1, thrombospondin), many cytokines and chemokines (e.g. transforming growth factor (TGF)- $\beta$, CXCL7, CXCL4, CXCL8, CCL5) and microbicidal effectors (e.g. thrombocidin 1 and 2, complement 3 precursor), which play an important role in the regulation of immune response [9, 11, 13-20]. Interestingly, $\alpha$-granules contain molecules that have opposite effects, such as pro- and anti-angiogenic molecules. Some observations suggest that these molecules are stored in different $\alpha$-granule subpopulations, and are differently released depending on the activating pathway $[1,11]$.

In addition, platelets express a high number of receptors and ligands that are found in other immune effectors and known to play a critical role at different stages of immune response. Several studies have shown that human and mouse platelets express functional Toll-like receptors (TLRs) including TLR1, 2, 4 and 6 on their surface, and TLR3, 7 and 9 in endosomes [1, 21-23]. TLRs are a family of pattern recognition receptors, expressed by various immune cells, especially cells of innate immunity (e.g. macrophages and dendritic cells). These receptors bind conserved molecular motifs expressed by pathogens (pathogen-associated molecular patterns) and endogenous danger signalling (damage-associated molecular patterns), and as a result they activate immune cells and promote the inflammatory response. It has been shown that platelet activation by specific oxidised phospholipids requires TLR2 and TLR6 [24], while the activation by lipopolysaccharide or carboxy(alkylpyrrole) protein adducts requires TLR4 [25] and TLR9 [26], respectively.

Furthermore, platelets express constitutively CD40 on their surface and its ligand CD40L (CD154) in their $\alpha$-granules, which is translocated to the platelet surface or secreted as soluble molecules upon activation $[27,28]$. The interaction between CD40 and CD40L plays a critical role in innate and adaptive immunity. Indeed, the activation of CD40 on the surface of antigen-presenting cells stimulates the secretion of inflammatory cytokines such as interleukin (IL)-1, IL-6 and tumour necrosis factor (TNF)- $\alpha$. In addition, the engagement of CD40 on B-cells by CD40L on T-cells promotes IgM to IgG class-switching [27, 29]. Platelet CD40 induces the formation of platelet-leukocyte aggregates, especially with monocytes, dendritic cells and neutrophils, but not with T-lymphocytes, and promotes the interaction between leukocytes and endothelial cells [28]. CD40L on activated platelets stimulates the upregulation of E-selectin, vascular cell adhesion protein-1 and ICAM-1 on the endothelial cell surface, molecules known to mediate the adhesion of immune cells to endothelium [27].

Siglecs (sialic acid-binding immunoglobulin-type lectins) are other immune-regulatory receptors found on platelets. Mainly expressed on haematopoietic cells, these cell membrane receptors are implicated in immune-response modulation. Two types of Siglec receptors have been described based on their inhibitory and activating functions. The principal duty of inhibitory Siglec is to recognise "self" sialic acids in order to reduce immune reactions $[21,30]$. Platelets express the inhibitory Siglec-7, -9 and -11 . The engagement of Siglec-7 by its specific ligand ganglioside GD2 induces platelet apoptosis, which may negatively regulate platelet inflammatory responses [21].

In addition, platelets carry other immune receptors such as complement receptors, the low-affinity immunoglobulin- $\gamma$ constant fragment region receptor II-a (Fc $\gamma$ RIIa), the high-affinity immunoglobulin- $\varepsilon$ constant fragment region receptor I (FceRI), CC- and CXC-chemokine receptors and triggering receptor expressed on myeloid cells 1 ligand [1, 9, 31].

Platelet immune molecules and receptors are presented in figure 2.

Platelets as actors in inflammation and immunity

In recent years, increasing number of studies has demonstrated that platelets are able to modulate the immune response through their interaction with immune cells in different pathological and physiological processes such as inflammation, tissue remodelling and autoimmune diseases [32-36]. The main plateletleukocyte interactions are summarised in table 1.

Platelets and innate immunity

Several studies have investigated the interaction between platelets and neutrophils and its impacts on the recruitment, adhesion, migration and activation of these cells. Indeed, multiple receptors and ligands are involved in the interplay between platelets and neutrophils [60, 61]. For example, the binding of platelets 


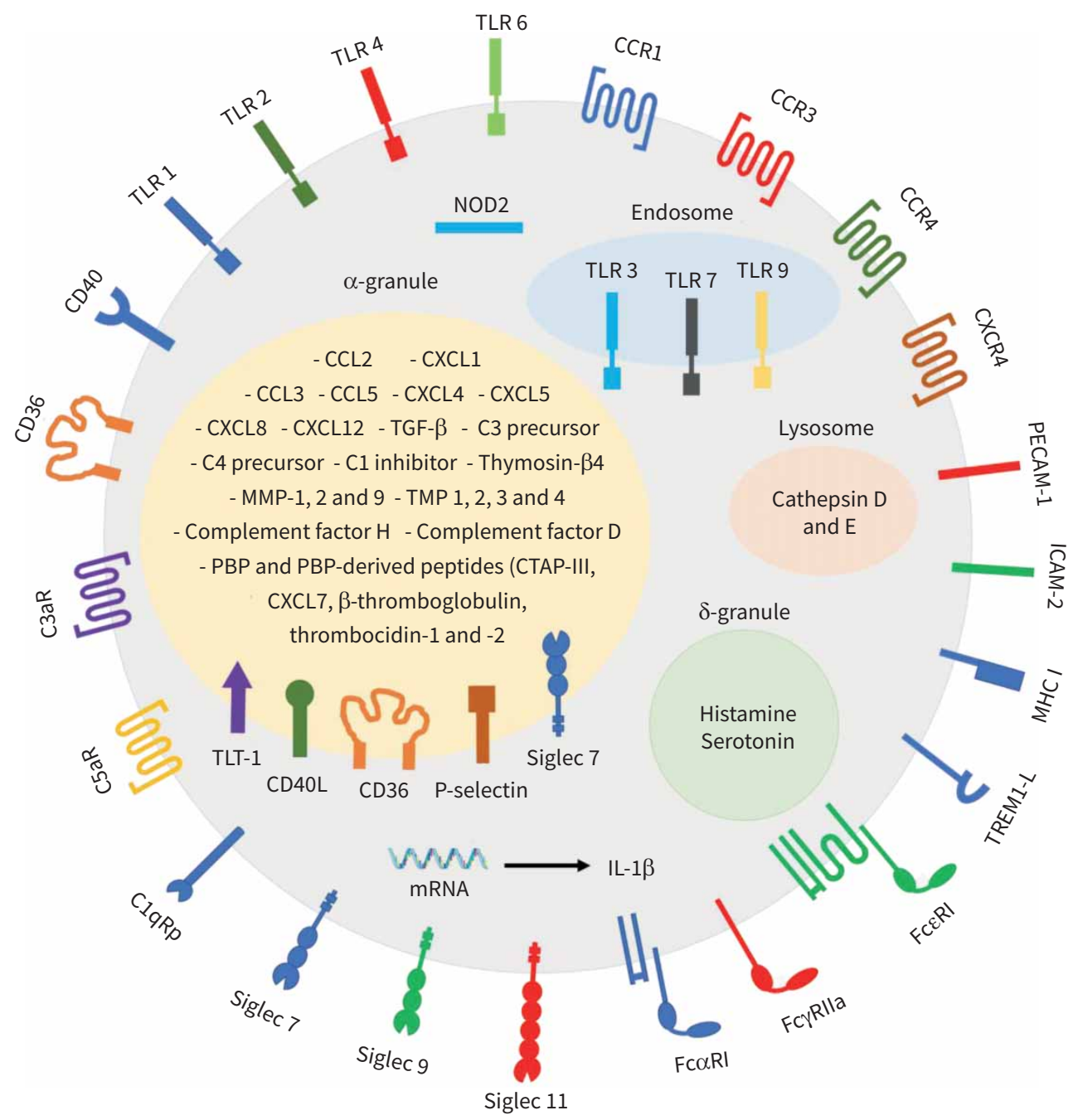

FIGURE 2 Platelet immune arsenal. TLR: Toll-like receptor; C3aR: complement 3a receptor; C5aR: complement 5a receptor; C1qRp: complement 1q receptor; Siglec: sialic acid-binding immunoglobulin-type lectin; FcoRI: immunoglobulin- $\alpha$ constant fragment region receptor I; FcyRlla: low-affinity immunoglobulin- $\gamma$ constant fragment region receptor II-a; FceRl: high-affinity immunoglobulin- $\varepsilon$ constant fragment region receptor I; TREM1-L: triggering receptor expressed on myeloid cells 1 ligand; MHC: major histocompatibility complex; ICAM: intercellular adhesion molecule; PECAM-1: platelet endothelial cell adhesion molecule 1; CCR: CC-chemokine receptors; CXCR: CXC-chemokine receptors; NOD: nucleotide-binding oligomerisation domain-containing protein; $\mathrm{CCL}$ : $\mathrm{CC}$-chemokine ligand; $\mathrm{CXCL}$ : $\mathrm{CXC}$-chemokine ligand; TGF: transforming growth factor; C: complement; MMP: matrix metalloproteinase; TIMP: tissue inhibitor of metalloproteinase; PBP: platelet basic protein; CTAP: connective tissue-activating peptide; TLT: TREM-like transcript; IL: interleukin.

P-selectin to neutrophil P-selectin glycoprotein ligand-1 promotes the adhesion of neutrophils to endothelial cells and enhance neutrophil transendothelial migration both in vivo and in vitro [37]. Neutrophils also enhance the adhesion of platelets by binding platelets directly or by unmasking platelet-binding sites on the inflamed vessel wall [38, 62]. Furthermore, incubation of neutrophils with platelets, in vitro, enhances neutrophil reactive oxygen species (ROS) generation in a CD40L-dependent manner [39]. In addition, stimulation of platelet TLR4 induces the activation of neutrophils and the release of neutrophil extracellular traps (NETs), enhancing bacterial trapping in septic blood [38].

Platelets interact with monocytes, leading to monocyte-platelet aggregation, and modulation of monocyte functions [40-43, 63-66]. Monocyte-platelet interaction induces a pro-inflammatory phenotype in circulating monocytes [65]. In addition, activated platelets stimulate the secretion of CCL2 and IL-8 (CXCL8) by monocytes in a P-selectin- and CCL5-dependent manner [40]. Monocytes stimulated by platelet factor 4 secrete a high amount of ROS which induces endothelial cell apoptosis [41]. It has also been shown that 
TABLE 1 Effects of platelet mediators on immune cells

Platelet mediators

\begin{tabular}{|c|c|c|c|c|}
\hline & Platelet mediators & Effect on leukocytes & Species & Reference \\
\hline \multicolumn{5}{|l|}{ Innate immunity } \\
\hline \multirow[t]{3}{*}{ Neutrophil } & P-selectin & $\begin{array}{c}\text { Promotes the adhesion of neutrophils to endothelial cells and enhances } \\
\text { neutrophil transendothelial migration }\end{array}$ & $\begin{array}{l}\text { Mouse } \\
\text { Human }\end{array}$ & {$[37]$} \\
\hline & TLR4 & Induces neutrophil activation and the release of NETs & Human & [38] \\
\hline & CD40L & Enhances neutrophil ROS generation & Human & [39] \\
\hline \multirow[t]{4}{*}{ Monocyte } & P-selectin and CCL5 & Stimulate monocyte secretion of MCP-1 and IL-8 & Human & [40] \\
\hline & PF4 & Enhances monocyte ROS generation & Human & [41] \\
\hline & & & Human & \\
\hline & CXCL12 & Supports monocyte differentiation towards $\mathrm{CD} 163^{+}$macrophages & Human & [43] \\
\hline \multirow[t]{2}{*}{ Macrophage } & $\begin{array}{l}\text { GPIb, GPVI and } \\
\text { thromboxane receptor }\end{array}$ & $\begin{array}{l}\text { Promote macrophage polarisation toward pro-inflammatory phenotype } \\
\text { and the release of pro-inflammatory mediators }\end{array}$ & $\begin{array}{l}\text { Mouse } \\
\text { Human }\end{array}$ & {$[44,45]$} \\
\hline & Prostaglandin E2 & Inhibits macrophage production of pro-inflammatory mediators & Mouse & [46] \\
\hline Dendritic cell & CD40L & Induces DC differentiation and maturation & Human & {$[47,48]$} \\
\hline Mast cell & PAF & Induces systemic mast cells activation and degranulation & $\begin{array}{l}\text { Mouse } \\
\text { Human }\end{array}$ & [55] \\
\hline \multicolumn{5}{|c|}{ Adaptive immunity } \\
\hline $\mathrm{CD}^{+}$T-cell & PF4, CCL5 and TGF- $\beta$ & $\begin{array}{c}\text { Enhance the differentiation of stimulated } \mathrm{CD}^{+} \text {T-cells into Th1, Th17 } \\
\text { and Treg }\end{array}$ & Human & [56] \\
\hline \multirow[t]{2}{*}{$\mathrm{CD}^{+} \mathrm{T}$-cell } & MHC class I & Activates $\mathrm{CD} 8^{+} \mathrm{T}$-cells & Mouse & [57] \\
\hline & CD40L & Enhance $\mathrm{CD} 8^{+} \mathrm{T}$-cell cytotoxic response & Mouse & {$[58]$} \\
\hline B-cell & CD40L & Induces isotype switching by B-cells & Mouse & {$[58,59]$} \\
\hline
\end{tabular}

NK: natural killer; TLR: Toll-like receptor; NETs: neutrophil extracellular traps; ROS: reactive oxygen species; MCP: monocyte chemoattractant protein; IL: interleukin; PF: platelet factor; GP: glycoprotein; DC: dendritic cell; TGF: transforming growth factor; GM-CSF: granulocyte-macrophage colony-stimulating factor; PAF: platelet-activating factor; Th: T-helper cell; Treg: regulatory T-cell; MHC: major histocompatibility complex.

platelets from patients with rheumatoid arthritis stimulate monocyte production of TNF- $\alpha$, IL-6 and matrix metalloproteinase-9 [66]. In contrast, activated platelets via the secretion of prostaglandin E2 can promote an anti-inflammatory phenotype in monocytes [42]. In addition, platelets support the differentiation of monocytes towards CD163 ${ }^{+}$macrophages, in a CXCL12- and CXCR4/CXCR7-dependent manner [43].

Additionally, platelets suppress the expression of anti-inflammatory markers by macrophages, through GPVI and thromboxane receptors, and promote the release of pro-inflammatory mediators such as IL-6 in mouse models of cutaneous inflammation [44]. In septic mice receiving platelet transfusions, platelets interact with macrophages via the GPIb-CD11b axis and promote the polarisation of these cells toward pro-inflammatory phenotype [45]. In vitro, the incubation of platelets with macrophages results in platelet phagocytosis and subsequent macrophage activation [67]. Moreover, platelet microparticles internalised by macrophages are able to modify the transcriptome of these cells, and enhance their phagocytic skills [68]. However, other studies have reported that platelets inhibit the production of pro-inflammatory mediators by macrophages $[46,69]$, and reprogramme the latter toward an anti-inflammatory phenotype producing IL-10 [42].

Platelets can affect dendritic cell (DC) functions. Supernatants from thrombin-activated platelets induce DC differentiation and maturation, as reflected by the expression of human leukocyte antigen-DR, the upregulation of CD83 and CD80 and the release of IL-12p40. This mechanism is dependent on soluble CD40L released by platelets [47, 48]. Moreover, the co-incubation of DCs with platelets enhances the ability of DCs to induce T-cell proliferation [49, 70]. Interestingly, in a mouse model of bacterial infection, platelets could deliver bacteria from circulation to spleen-resident DCs, in order to stimulate a cytotoxic $\mathrm{CD}^{+} \mathrm{T}$-cell response [71].

Additionally, platelets may impair the function of natural killer (NK) cells, known for their cytotoxic activity against infected or transformed cells, without the need for prior sensitisation [50]. Indeed, formation of platelet aggregates around tumour cells were found to be protective from NK cell cytotoxicity 
by generating a physical barrier between tumour cells and NK cells [72]. Moreover, platelet-derived TGF- $\beta$ may suppress the expression of NK cell activating receptors, such as natural killer group 2 member $\mathrm{D}$, and inhibit NK cell degranulation and target cell lysis [50, 51, 52].

Platelets have also been suggested to promote eosinophilia. In vitro, platelets enhance eosinophil survival through the release of granulocyte-macrophage colony-stimulating factor, even in the presence of molecules that promote apoptosis [53]. Additionally, in a murine model of allergic inflammation, platelets bind to eosinophils through P-selectin, and promote their entry into the lung, probably by enhancing their expression of adhesion molecules [54]. Furthermore, following an endothelial injury in murine model of atherosclerosis, eosinophils get in direct contact with platelets at the site of injury, which results in eosinophil activation and eosinophil extracellular traps secretion [73].

The interplay between platelets and mast cells or basophils has been only rarely studied. Recent work in mice has demonstrated that systemic platelet activation causes platelet aggregation on blood vessels in close proximity to perivascular mast cells, and the release of platelet-activating factor (PAF). In turn, PAF induces systemic mast cell activation and degranulation, which result in shock (characterised mainly by sharp drop in core body temperature), vascular leakage and tissue inflammation [55].

\section{Platelets and adaptive immunity}

In addition to platelet abilities in modulating antigen-presenting cells, which in turn stimulate the adaptive immune response, platelets can interact directly with T- and B-lymphocytes. Under arterial flow conditions, platelets increase the adhesion of lymphocytes to collagen-coated surfaces and adhered lymphocytes are usually seen in association with platelets and platelet microaggregates [74]. Moreover, platelets enhance the differentiation of stimulated $\mathrm{CD}^{+}$T-cells into type 1 T-helper (Th1) cells, Th17 and regulatory T-cells (Treg), which is reflected by increased production of interferon (IFN)- $\gamma$, IL-17 and IL-10, respectively. These platelet-enhancing effects are mediated both via direct cell-to-cell contact and via the release of soluble mediators including platelet factor (PF)4, CCL5 and TGF- $\beta$ [56]. Furthermore, platelet microparticles, through their associated TGF- $\beta$ also induce the differentiation of stimulated CD4 ${ }^{+}$T-cells into functional Tregs [75]. Interestingly, platelets are capable of processing plasmodium-derived antigen, thereby activating $\mathrm{CD}^{+}$T-cells in a MHC class I-dependent manner both in vitro and in vivo [57]. Furthermore, the transfusion of wild-type or $\mathrm{CD}_{40 \mathrm{~L}^{-1-}}$ platelets into $\mathrm{CD}_{0} \mathrm{~L}^{-/-}$mice infected with adenovirus, provides evidence that platelets induce isotype switching by B-cells and enhance $\mathrm{CD}^{+} \mathrm{T}$-cell cytotoxic response, through CD40L [58]. Likewise, membrane vesicles (microparticles and/or exosomes) derived from wild-type, but not $\mathrm{CD}_{40 \mathrm{~L}^{-1-}}$ platelets, increase the production of adenovirus-specific IgG and enhance germinal centre formation in infected CD40L $\mathrm{L}^{-1-}$ mice [59].

\section{Platelets as pathogen sensors}

Platelets can detect and respond to different pathogens such as bacteria, viruses, parasites or fungi. Platelets, through different receptors, can interact directly with pathogens and their secreted products or indirectly by binding plasma proteins adhered to pathogens [76-80]. For instance, Staphylococcus aureus can bind to platelet GPIIb/IIIa, either directly or indirectly through fibrinogen and fibronectin [81, 82]. Cytomegalovirus and HIV-1 can bind platelet TLR2 and C-type lectin-like receptor-2, respectively [83, 84]. Usually, the interaction between platelets and pathogens induces platelet activation and the release of different antimicrobial molecules such as platelet microbicidal peptides, thrombocidins and kinocidins [76-80]. Platelets incubated with $S$. aureus impair the growth of this pathogen by the release of $\beta$-defensin 1 [85]. In addition, platelets, through the release of PF4, inhibit HIV-1 infection of T-cells at the stage of virus attachment and entry [86, 87] and are able to kill Plasmodium parasites [88, 89]. Furthermore, platelet-derived PF4, CCL5 and thrombocidin 1 exert antifungal activity against Candida by inhibiting its growth [79]. Beside their direct effect on pathogens, platelets interact with other immune cells and thereby modulate the infectious immune response [76-80]. For instance, $\beta$-defensin 1 released by platelets in response to $S$. aureus induces NET release by neutrophils [85]. Interestingly, in mice infected with Listeria monocytogenes, platelets bind the micro-organism in the bloodstream and deliver it to splenic $\mathrm{CD}^{+} \mathrm{DCs}$, which in turn induce the CD8 ${ }^{+} \mathrm{T}$-cell response [71].

\section{Platelets in lung pathology}

\section{Platelets in pulmonary infectious diseases}

The main bacterial cause of community-acquired pneumonia and sepsis is Streptococcus pneumoniae, which directly activates human and mouse platelets in vitro [90, 91]. In vivo, mouse models highlight the involvement of platelets in the control of bacterial infections. Indeed, thrombocytopenic mice infected with Strep. pneumoniae [92], Klebsiella pneumoniae or Pseudomonas aeruginosa [93-95] showed increased bacterial dissemination, systemic inflammation and mortality when compared to control mice. Moreover, 
platelets were detected at the site of infection in a mouse model of tuberculosis and platelet-associated molecules such as platelet-derived growth factor (PDGF)-BB were upregulated in plasma and bronchoalveolar lavage (BAL) fluid of patients with tuberculosis [96].

In patients infected with influenza A virus subtype H1N1 (A/H1N1), platelet-monocyte aggregates are increased compared to control subjects [97]. A/H1N1 activates platelets and triggers the expression of surface receptor such as P-selectin and the release of platelet microparticles [98]. In addition, A/H1N1 induces the release of C3 from platelets, in a TLR7-dependent manner, which promotes platelet-neutrophil aggregation and the release of NETs [99]. Interestingly, a study in influenza-infected mice suggested that, in contrast to the protective role of platelets in bacterial pneumonia models, these cells may enhance pulmonary damage in influenza pneumonia. Indeed, platelet-neutrophil aggregates strongly increase in intra-alveolar lung air spaces and pulmonary vasculature of infected mice, and platelet-associated neutrophils release large quantities of NETs. Treatment of influenza-infected mice with both clopidogrel (platelet inhibitor) and oseltamivir (antiviral agent) reduces neutrophil-platelet aggregates, NET release and pulmonary damage and improves mouse survival when compared to oseltamivir alone [100]. These results emphasise the contribution of platelets to influenza pathology and lung damage. Studies have been conducted in patients infected with severe acute respiratory syndrome coronavirus 2 (SARS-CoV-2), the newly discovered strain of coronavirus causing the ongoing coronavirus disease 2019 (COVID-19) pandemic. COVID-19 is associated with increased risk of thrombotic events, particularly in patients who require intensive care. Platelet-leukocyte aggregation and P-selectin expression on platelet surfaces are increased in COVID-19 patients compared to control subjects. In addition, platelets from SARS-CoV-2 infected patients had hyperreactive responses to agonists, higher spreading on fibrinogen and collagen and faster aggregation. Finally, platelet transcriptome profiling revealed significant changes in the gene expression profile of platelets from COVID-19 patients, including changes in immune pathways [101]. Despite reports indicating an alteration in platelet activation state and function in COVID-19, it is still not clear how platelets contribute to thrombotic complications in this disease. A recent study [102] showed that, compared to control groups, platelets from patients with severe COVID-19 have an upregulation of apoptotic markers including phosphatidylserine externalisation, a molecule involved in blood coagulation, and that platelet apoptosis markers correlate with plasma levels of D-dimer. In addition, COVID-19 patients with thromboembolic complications had significantly higher phosphatidylserine externalisation compared with those without thrombosis. Interestingly, sera and IgG fractions isolated from COVID-19 patients were able to induce apoptosis in platelets from healthy donors, and neutralisation of FcyRIIA receptors inhibited antibody-mediated platelet apoptosis. These observations suggest that IgG in severe COVID-19 patients might induce platelet apoptosis which leads to phosphatidylserine surface exposition and enhancement of coagulation reactions. However, further studies are needed in order to confirm this concept and to fully elucidate the role of platelet in the physiopathology of COVID-19 [102]. Furthermore, platelets might be involved in the response to respiratory syncytial virus (RSV), which is the most common cause of lower respiratory tract infections in young children and immunecompromised patients. Whole-blood transcriptomic profile of infants infected with RSV showed significant overexpression of platelet-related genes [103]. In vitro, platelets defend monocytes against RSV infections by internalising viral particles and by enhancing type I IFN production from leukocytes [104].

Platelets have been shown to play a protective role against aspergillosis, which is the most common mould infection worldwide, caused mainly by Aspergillus fumigatus [105-107]. In vitro, platelets interact with both conidia and hyphae of $A$. fumigatus, which results in platelet activation. In turn, platelets damage $A$. fumigatus hyphae, as shown by the loss of cell wall integrity, and decrease fungal germination, hyphal elongation and colony size [105-107].

Additionally, platelets, probably by PF4 release, are able to kill all major species of Plasmodium parasites responsible for malaria disease [88, 89], which in its severe form, may cause pulmonary complications such as acute respiratory distress syndrome [108].

Therefore, platelets may either be involved in host defence or tissue damage, depending on the pathogen.

\section{Platelets in asthma and other chronic respiratory diseases}

Asthma is a chronic inflammatory disease characterised by bronchial hyperresponsiveness to nonspecific stimuli, immune cell infiltration to airway tissues, airway remodelling and mucus overproduction [109]. Asthma is a heterogeneous disease with different phenotypes and endotypes, the latter defined by distinct pathophysiological and immunological features. Two main endotypes have been described: the T2-high and the T2-low endotypes. The T2-high endotype is orchestrated by several cytokines produced by the Th2-cells and type 2 innate lymphoid cells, namely IL-4, IL-5 and IL-13, associated with eosinophilic inflammation 
[110, 111]. The T2-low endotype is less known, and mostly defined by a low presence or absence of T2-mediated inflammation markers. This endotype includes neutrophilic inflammation orchestrated by Th1 and Th17 cytokines such as IFN- $\gamma$ and IL-17, respectively, mixed eosinophilic and neutrophilic inflammation, and paucigranulocytic inflammation in which few granulocytes are involved [110, 111].

Several studies have suggested the implication of platelets in asthma. In vivo, the challenge of allergic asthma patients with the allergen Dermatophagoides pteronyssinus resulted in peripheral blood platelet activation. This activation is reflected by an increase in plasma level of PF4, $\beta$-thromboglobulin and soluble P-selectin [112]. In addition, the amount of circulating platelet microparticles is significantly higher in asthmatic patients compared to nonasthmatic subjects [113], and platelets from allergic asthma patients have elevated brain-derived neurotrophic factor (BDNF) concentrations [114]. BDNF is a mediator of neuronal plasticity that contributes to airway obstruction and hyperresponsiveness. In patients without inhaled corticosteroid treatment, enhanced platelet BDNF concentrations are correlated with airflow limitation and airway hyperresponsiveness [114]. In addition to platelet activation markers found in the blood, the levels of PF4 and $\beta$-thromboglobulin increase significantly in the BAL of allergic asthma patients, which indicates platelet activation in patient airways [115]. In vitro, human IgE and anti-human IgE stimulation of platelets showed higher amount of CCL5 released by platelets from allergic asthma patients than platelets from healthy individuals [116]. Moreover, platelets bound to eosinophils of patients with allergic asthma promote the adhesion of these eosinophils to TNF- $\alpha$ activated endothelial cells, under flow conditions [117]. Additionally, platelets from allergic patients have reduced aggregation response to conventional stimuli (i.e. ADP and collagen). This platelet phenotype is thought to be a consequence of chronic in vivo activation, leading to platelet functional "exhaustion" [118-121]. A bioenergetic analysis of platelets from asthmatic and healthy individuals showed that platelets from asthmatic patients had increased tricarboxylic acid cycle enzymatic activity (which fuels oxidative phosphorylation) and rely less on glucose and glycolysis for cellular respiration [122]. In patients with severe food-associated respiratory allergy, transcriptomic analyses on peripheral blood mononuclear cells showed a downregulation of transcripts involved in platelet adhesion, activation, shape change, granule secretion, aggregation and other platelet functions [123]. In this context, transcriptome profiling of platelets from asthmatic patients may provide additional knowledge on platelet altered function in asthma.

Aspirin-exacerbated respiratory disease (AERD) is a chronic inflammatory disease characterised by the clinical triad of asthma, nasal polyposis and respiratory reactions to aspirin and other nonsteroidal anti-inflammatory drugs. Platelet activation markers, such as P-selectin and CD63, are overexpressed in patients with AERD compared to control subjects [124]. Moreover, circulating neutrophils, eosinophils and monocytes with adherent platelets on their surfaces are found in higher proportions in AERD patients than in aspirin-tolerant controls. In addition, platelets colocalised with leukocytes in nasal polyps of AERD patients [125].

In addition to research in humans, several studies were conducted in mouse models of allergic asthma, providing more evidence of platelet involvement in the disease. The main findings are summarised in figure 3. In ovalbumin (OVA)-sensitised mice, platelets alone or associated to leukocytes, migrate out of blood vessels into lung tissues after OVA challenge in an FceRI-dependent mechanism [126]. The percentage of platelets with IgE bound to their surface is significantly higher in OVA-sensitised mice compared to sham-sensitised mice. Moreover, platelets from sensitised wild-type mice but not from sensitised $\mathrm{FcRy}^{-1-}$ mice (which lack FceRI) are able to migrate toward the sensitising allergen or an anti-IgE antibody in vitro. These observations suggest that upon inhalation, allergens may induce cross-linking of contiguous platelet FceRI through IgE, and favour subsequent platelet migration to lung tissues of sensitised mice [126]. Platelet migration to allergic mouse lung was described in a more recent study showing that platelet recruitment occurs via a CCR3-dependent mechanism. Once in lung tissue, platelet could release mediators involved in the exacerbation of asthma, such as histamine and serotonin, that induce bronchoconstriction and pro-inflammatory cytokines that enhance local immune cell activation and pulmonary recruitment of further circulating immune cells. Additionally, platelets can release a number of growth factors implicated in tissue remodelling and angiogenesis. Conversely, platelets, through other secreted mediators, may participate to tissue repair and inflammatory response resolution. So far, to our knowledge, no study has investigated the exact role of platelets in lung tissue and its significance in asthma pathology [127]. In OVA-sensitised and challenged mice, circulating platelet-leukocyte complexes are increased, and platelet-associated leukocytes exhibit elevated expression of the adhesion molecule CD11b [54]. In parallel, the recruitment of leukocytes in lung tissues requires the expression of P-selectin and platelet P2Y1 receptor on platelet surfaces [54, 128]. Platelets were found to release myosin light-chain 9/12 (Myl9/12) in the pulmonary circulation of OVA-challenged mice. Then, Myl9/12 attaches to the luminal surface of blood vessels, form net-like structures inside the vessels and binds to CD69 expressed by activated leukocytes, thereby facilitating the recruitment of these leukocytes in lung tissues [129]. 


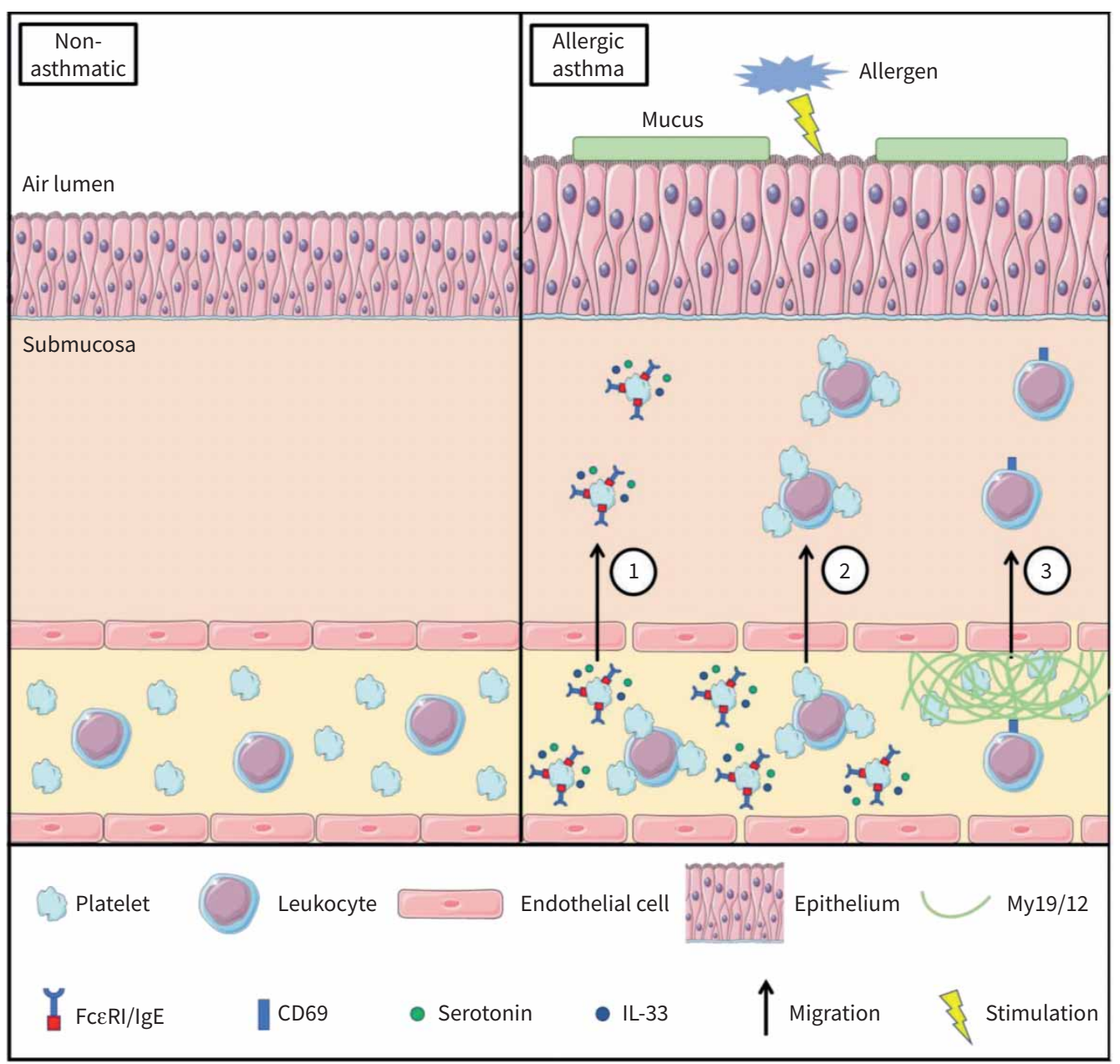

FIGURE 3 Hypothetical platelet functions in mouse models of allergic asthma. In mouse models of allergic asthma: 1) platelets bind IgEs, release serotonin and interleukin (IL)-33 which promote airway inflammation, and migrate out of blood vessels to inflamed lung tissues; 2) platelets aggregate with leukocytes and promote their migration to the lung, in a mechanism implicating platelet P-selectin, CD40L, high-affinity

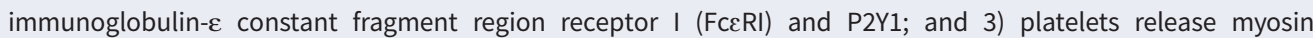
light-chain (My) $19 / 12$, which binds to blood vessels and forms net-like structures inside the vessels. Myl9/12 interacts with CD69 on activated leukocytes, thereby facilitating their recruitment to lung tissues.

Moreover, platelets appear to play a critical role in the progression of experimental allergic asthma through CD40L [130]. The administration of platelets from wild-type but not CD40L ${ }^{-/-}$mice to OVA-sensitised mice enhances pulmonary inflammation, serum OVA-specific IgE levels and lung IL-4 and IL-13 expression, but inhibits Treg generation [130]. In addition, platelet depletion in mice repeatedly exposed to OVA, reduces epithelial and smooth-muscle thickening and subepithelial fibre deposition in the airways when compared with control mice, suggesting the involvement of platelets in airway remodelling [131]. Another study reveals that in addition to their involvement in the late exposure phase of allergic asthma, platelets play an important role in the initial sensitisation phase of the disease, as their temporary depletion during the initial OVA sensitisation step suppressed allergic asthma features upon subsequent allergen exposure [132]. Furthermore, OVA-sensitised $\mathrm{TPH}^{-1-}$ mice (which lack serotonin) showed reduced airway inflammation upon OVA exposure, compared to OVA-sensitised wild-type mice. The transfusion of wild-type platelets but not THP1 $1^{-/}$platelets to OVA-sensitised $\mathrm{TPH} 1^{-/-}$mice restores all allergic airway inflammation hallmarks, suggesting a key role of platelet-derived serotonin in the disease [133]. Finally, it has been shown that platelets express constitutive IL-33 protein and that platelet-derived IL-33 promote papain-induced airway eosinophilic inflammation in mice [134].

Other animal models provide further evidence supporting platelet involvement in asthma. In allergic rabbit models, platelet depletion reduces the development of IgE-induced anaphylactic shock and inhibits 
bronchial hyperresponsiveness in response to histamine. Additionally, it has been shown that thromboxane A2 is essential for the induction of bronchoconstriction in sensitised guinea pigs. Moreover, platelet depletion in these animals significantly reduces thromboxane A2 levels in the plasma and antigen-induced bronchoconstriction, suggesting that platelets are the major source of thromboxane A2 in this model [118].

Few studies have been conducted in order to investigate the role of platelets in chronic inflammatory respiratory diseases other than asthma. In patients with stable COPD, the amounts of platelet microparticles and platelet-monocyte aggregates are higher than in healthy subjects, and even more so during an acute exacerbation of the disease $[135,136]$. In addition, thrombocytosis was associated with increased shortand long-term mortality after COPD exacerbation, while antiplatelet therapy was associated with reduced 1-year mortality [137]. Proteomic analysis of lung tissues from COPD patients showed a downregulation of proteins related to platelet activation such as PF4, GPVI and thrombospondin-1 [138]. Moreover, in chronic smoke-exposed animals with COPD-like lung lesions, circulating platelets showed mitochondrial functioning abnormalities [139].

In patients with idiopathic pulmonary fibrosis (IPF), the percentage of platelet-monocyte aggregates is also elevated when compared to non-IPF controls [140]. The stimulation of platelets from IPF patients with different agonists showed increased activation compared to platelets from control subjects. This hyperactivity is probably due to the plasma environment, as incubation of control platelets in plasma from IPF patients increased platelet reactivity to agonists compared to control plasma [141]. Proteomic analysis of peripheral blood taken from IPF patients or normal controls showed an upregulation of proteins related to platelet activation in the blood of IPF patients [142]. Furthermore, in a mouse model of pulmonary fibrosis, the administration of cangrelor (a well-known antiplatelet agent) decreases platelet-neutrophil aggregates and neutrophil infiltration, thereby reducing lung inflammation and pulmonary fibrosis [143].

Patients with chronic thromboembolic pulmonary hypertension (CTEPH) exhibit platelet abnormalities compared to control subjects, such as decreased platelet count, higher spontaneous platelet aggregation but decreased platelet aggregation induced by standard concentrations of different agonists [144]. In addition, platelets from CTEPH patients showed increased surface P-selectin and activated GPIIb/IIIa expression at baseline and after stimulation with thrombin [145]. Moreover, platelet-derived microparticle levels are elevated in CTEPH patient blood and correlate with right atrial pressure and cardiac index [146]. Platelet numbers and activation state are also altered in patients with acute respiratory distress syndrome [147, 148].

The evidence for platelet involvement in lung chronic diseases is summarised in table 2.

These observations suggest that platelets could play an important role in various lung diseases, hence further studies are needed in order to elucidate the precise mechanisms involving platelets in the physiopathology of these pathologies.

\section{Antiplatelet therapy in chronic respiratory diseases}

Several clinical studies have evaluated the effect of antiplatelet therapy in chronic respiratory diseases, especially aspirin and P2Y12 receptor antagonists (prasugrel, clopidogrel and ticlopidine). In patients with persistent asthma, neither aspirin nor prasugrel treatments improved airway inflammation, pulmonary function and asthma symptoms [149]. In contrast, in patients with COPD, antiplatelet treatments exhibited many beneficial effects on the course of the disease. The progression of "percentage emphysema" was slower in COPD patients who used aspirin regularly compared to those who did not, and regular aspirin use was correlated with significant reduction in the rate of emphysema progression over 10 years. Moreover, aspirin was associated with a significantly improved quality of life and lower odds of dyspnoea development in COPD patients, and lower risk of in-hospital death and need for invasive mechanical ventilation in patients hospitalised for acute exacerbation. Finally, a meta-analysis suggested that antiplatelet treatment might significantly reduce all-cause mortality in COPD patients (reviewed in [150]). These clinical studies do not allow the discrimination of the mechanisms involved in the improvement of COPD patients.

All these clinical trials focused on drugs commonly used in the secondary prevention of arterial thrombosis. However, an increasing number of observations suggest that platelet activation in some inflammatory contexts may be different from platelet activation during haemostasis. For instance, despite the well-known antithrombotic effects of aspirin, this drug does not attenuate platelet-leukocyte interaction [151]. In addition, while platelet activation markers are detected in patients with allergic diseases, only mild haemostatic defects have been reported and the incidence of cardiovascular events in these patients is not greater than in the general population [121, 149]. Interestingly, platelets activation with TLR7 agonists 


\begin{tabular}{|c|c|c|}
\hline \multicolumn{3}{|l|}{ Asthma } \\
\hline $\begin{array}{l}\text { Circulating platelet activation following the challenge of allergic asthma patients with the allergen Dermatophagoides } \\
\text { pteronyssinus }\end{array}$ & Human & [112] \\
\hline High amounts of platelet-derived microparticles in blood & Human & [113] \\
\hline Increased levels of platelet activation markers in BAL following antigen challenge & Human & [115] \\
\hline Platelet migration into lung tissues of OVA-sensitised mice after OVA challenge & Mouse & [126] \\
\hline $\begin{array}{l}\text { The recruitment of leukocytes to the lung tissues of OVA-sensitised mice requires platelet P-selectin and P2Y1 } \\
\text { platelet receptor }\end{array}$ & Mouse & {$[54,128]$} \\
\hline $\begin{array}{l}\text { Reduced epithelial and smooth-muscle thickening and subepithelial fibre deposition in the airways of OVA-sensitised mice } \\
\text { depleted of platelets }\end{array}$ & Mouse & [131] \\
\hline Platelet derived IL-33 promotes papain-induced airway eosinophilic inflammation & Mouse & [134] \\
\hline \multicolumn{3}{|l|}{ COPD } \\
\hline High amounts of circulating platelet microparticles and platelet-monocyte aggregates & Human & {$[135,136]$} \\
\hline Association between thrombocytosis and increased short- and long-term mortality after COPD exacerbation & Human & [137] \\
\hline Downregulation of proteins related to platelet activation in lung tissues & Human & [138] \\
\hline Platelets abnormalities in mitochondrial functioning & Mouse & [139] \\
\hline \multicolumn{3}{|l|}{ IPF } \\
\hline High amount of circulating platelet-monocyte aggregates & Human & [140] \\
\hline Platelets hyperactivity toward diverse agonists & Human & [141] \\
\hline Upregulation of proteins related to platelet activation in blood & Human & [142] \\
\hline $\begin{array}{l}\text { Reduced lung inflammation and pulmonary fibrosis following the administration of an antiplatelet agent in a mouse } \\
\text { model of pulmonary fibrosis }\end{array}$ & Mouse & [143] \\
\hline \multicolumn{3}{|l|}{ CTEPH } \\
\hline Decreased platelet count but elevated platelet-derived microparticle levels in blood & Human & {$[144,146]$} \\
\hline Increased platelet activation markers & Human & [145] \\
\hline \multicolumn{3}{|l|}{ ARDS } \\
\hline Altered platelet numbers and platelet activation state & Human & {$[147,148]$} \\
\hline
\end{tabular}

BAL: bronchoalveolar lavage; OVA: ovalbumin; IL: interleukin; IPF: idiopathic pulmonary fibrosis; CTEPH: chronic thromboembolic pulmonary hypertension; ARDS: acute respiratory distress syndrome.

does not promote thrombotic behaviour such as platelet aggregation, but increases the interaction of platelets with leukocytes. TLR7 stimulation induces the formation of small groups of platelets which extend some pseudopodia-like structures. These changes were markedly different to those observed following platelet activation with thrombin, where platelets completely lose their discoid shape and extend excessive pseudopodia-like structures, which results in tight clot formation [152]. Furthermore, in platelets stimulated by P2Y1 agonists, specific inhibition of phospholipase C suppressed platelet aggregation, but not platelet chemotaxis toward $N$-formyl-methionyl-leucyl-phenylalanine or platelet-induced neutrophil chemotaxis toward macrophage-derived chemokine. In contrast, the inhibition of the GTPase Rac1 and the kinase ROCK failed to inhibit platelet aggregation, but was able to attenuate platelet chemotaxis and platelet-induced neutrophil chemotaxis, suggesting differential downstream signalling pathways of platelet P2Y1 purinergic receptor [149]. However, Rac1 was shown to play either minor or central role in platelet aggregation [153], emphasising that more studies are needed to fully understand platelet activation pathways. Another study in a mouse model of allergic asthma also supports the involvement of P2Y1 signalling in platelet inflammatory response. In this model, thrombocytopenia significantly reduced leukocyte recruitment to the lung after allergen challenge. Pulmonary leukocyte recruitment was restored in thrombocytopenic mice reinfused with washed platelets, whereas it remained suppressed after the infusion of platelets pre-treated with P2Y1 antagonist or Rho-associated kinase 1 inhibitor. These observations suggest that, following P2Y1 stimulation, RhoA signalling in platelets controls leukocyte recruitment to lung tissues of allergic mice [128].

Hence, a better understanding of platelet activation mechanisms in different stimulatory and pathophysiological conditions might lead to the development of new drugs that target platelet inflammatory activities without necessarily affecting the thrombotic functions.

In addition, little is known about the effects of respiratory treatments on platelets, either classical therapies (such as $\beta$-adrenergic receptor agonist and glucocorticoids), or newer biotherapies used in the treatment of asthma or COPD. It has been shown that human platelets exhibit functional glucocorticoid receptors [154]. In vitro, prednisolone inhibited platelet aggregation and platelet-monocyte interaction [154, 155]. The new 
biologics used in severe asthma have some side-effects including decreased production of platelets, shortened platelet lifespan or sequestration in vascular beds which result in thrombocytopenia [156]. However, platelet activation could also be modified by therapeutics targeting IgE, thymic stromal lymphopoietin (TSLP), IL-33, IL-5 or IL-4/IL-13. For example, therapeutics targeting IgE or TSLP may act directly on platelets that express receptors of these molecules [116, 157]. In contrast, targeting IL-5 may indirectly reduce platelet activation through depletion of eosinophils, shown to activate platelets through major basic protein release [154, 155].

\section{Conclusion}

Since their discovery, platelets have long been known as key players in haemostasis and thrombosis. However, accumulating evidence reveals that these anucleate cells are also professional modulators of inflammatory reactions, thanks to their ability to express distinct immune receptors and mediators, which allow them to interact with different immune cells. Platelets express both inflammatory and anti-inflammatory modulators and the exact mechanisms that allow platelets to stimulate either response are still unknown. In addition, platelets might be implicated in several airway diseases as particularly reflected by platelet activation markers in patient's circulation. Yet, a lot of work is still needed in order to determine the specific role of platelets in the pathophysiology of these diseases. Antiplatelet therapy using aspirin and P2Y12 antagonists may have beneficial effects in some airway diseases with high risk of comorbidity and cardiovascular events, such as COPD. However, several observations suggest that platelet activation in some inflammatory contexts may be different from platelet activation during haemostasis and thrombosis. This could explain why aspirin and anti-P2Y12 therapies failed to improve the management of some airway diseases, although platelet activation markers are detected in affected patients. Therefore, a better understanding of distinct platelet activation pathways may open up the possibility of finding novel therapeutic approaches targeting platelet inflammatory activities. Finally, it is important to notice that the highly sensitive nature of platelets, their size and the absence of nucleus make them one of the hardest cells to manipulate and a difficult subject of research. Classical purification methods of platelets generally lead to a very weak contamination by leukocytes and red blood cells. However, it has been estimated that leukocytes contain 12500-fold more mRNAs and 65-fold more proteins than platelets [158, 159]. In addition, platelets are usually used in relatively high concentrations, thereby increasing the number of contaminating cells. Consequently, results may be significantly altered, especially in omics studies and co-culture experiments. Therefore, serious caution must be taken when considering platelet purification and result interpretations.

Provenance: Submitted article, peer reviewed

Conflict of interest: M. Chebbo has nothing to disclose. C. Duez has nothing to disclose. M.C. Alessi has nothing to disclose. P. Chanez reports receiving grants or contracts, consulting fees, payment or honoraria for lectures, presentations, speakers' bureaus, manuscript writing or educational events, support for attending meetings and/or travel fees, in the past 36 months from: ALK, AstraZeneca, Boehringer Ingelheim, Chiesi and Novartis, outside the submitted works. D. Gras has nothing to disclose.

\section{References}

1 Semple JW, Italiano JE, Freedman J. Platelets and the immune continuum. Nat Rev Immunol 2011; 11: 264-274.

2 Hou Y, Carrim N, Wang Y, et al. Platelets in hemostasis and thrombosis: novel mechanisms of fibrinogen-independent platelet aggregation and fibronectin mediated protein wave of hemostasis. J Biomed Res 2015; 29: 437-444.

3 Machlus KR, Italiano JE. The incredible journey: from megakaryocyte development to platelet formation. J Cell Biol 2013; 201: 785-796.

4 Thon JN, Montalvo A, Patel-Hett S, et al. Cytoskeletal mechanics of proplatelet maturation and platelet release. J Cell Biol 2010; 191: 861-874.

5 Koupenova M, Clancy L, Corkrey HA, et al. Circulating platelets as mediators of immunity, inflammation, and thrombosis. Circ Res 2018; 122: 337-351.

$6 \quad$ Łukasik ZM, Makowski M, Makowska JS. From blood coagulation to innate and adaptive immunity: the role of platelets in the physiology and pathology of autoimmune disorders. Rheumatol Int 2018; 38: 959-974.

7 Holinstat M. Normal platelet function. Cancer Metastasis Rev 2017; 36: 195-198.

8 Pariser DN, Hilt ZT, Ture SK, et al. Lung megakaryocytes are immune modulatory cells. J Clin Invest 2021; 131: e137377.

9 Li JL, Zarbock A, Hidalgo A. Platelets as autonomous drones for hemostatic and immune surveillance. J Exp Med 2017; 214: 2193-2204. 
Lefrançais E, Ortiz-Muñoz G, Caudrillier A, et al. The lung is a site of platelet biogenesis and a reservoir for haematopoietic progenitors. Nature 2017; 544: 105-109.

Blair P, Flaumenhaft R. Platelet $\alpha$-granules: basic biology and clinical correlates. Blood Rev 2009; 23 177-189.

Golebiewska EM, Poole AW. Platelet secretion: from haemostasis to wound healing and beyond. Blood Rev 2015; 29: 153-162.

Weyrich AS, Zimmerman GA. Platelets: signaling cells in the immune continuum. Trends Immunol 2004; 25 : 489-495.

Karshovska E, Weber C, von Hundelshausen P. Platelet chemokines in health and disease. Thromb Haemost 2013; 110: 894-902.

Boehlen F, Clemetson KJ. Platelet chemokines and their receptors: what is their relevance to platelet storage and transfusion practice? Transfus Med 2001; 11: 403-417.

Burkhart JM, Gambaryan S, Watson SP, et al. What can proteomics tell us about platelets? Circ Res 2014; 114: 1204-1219.

Wijten P, van Holten T, Woo LL, et al. High precision platelet releasate definition by quantitative reversed protein profiling - brief report. Arterioscler Thromb Vasc Biol 2013; 33: 1635-1638.

Burkhart JM, Vaudel M, Gambaryan S, et al. The first comprehensive and quantitative analysis of human platelet protein composition allows the comparative analysis of structural and functional pathways. Blood 2012; 120: e73-e82.

Maynard DM, Heijnen HFG, Horne MK, et al. Proteomic analysis of platelet $\alpha$-granules using mass spectrometry. J Thromb Haemost 2007; 5: 1945-1955.

Maynard DM, Heijnen HFG, Gahl WA, et al. The $\alpha$-granule proteome: novel proteins in normal and ghost granules in gray platelet syndrome. J Thromb Haemost 2010; 8: 1786-1796.

Cognasse F, Nguyen KA, Damien P, et al. The inflammatory role of platelets via their TLRs and Siglec receptors. Front Immunol 2015; 6: 83.

Koupenova M, Mick E, Mikhalev E, et al. Sex differences in platelet toll-like receptors and their association with cardiovascular risk factors. Arterioscler Thromb Vasc Biol 2015; 35: 1030-1037.

Dib PRB, Quirino-Teixeira AC, Merij LB, et al. Innate immune receptors in platelets and platelet-leukocyte interactions. J Leukoc Biol 2020; 108: 1157-1182.

Biswas S, Zimman A, Gao D, et al. TLR2 plays a key role in platelet hyperreactivity and accelerated thrombosis associated with hyperlipidemia. Circ Res 2017; 121: 951-962.

Zhang G, Han J, Welch EJ, et al. Lipopolysaccharide stimulates platelet secretion and potentiates platelet aggregation via TLR4/MyD88 and the cGMP-dependent protein kinase pathway. J Immunol 2009; 182: 7997-8004.

Panigrahi S, Ma Y, Hong L, et al. Engagement of platelet toll-like receptor 9 by novel endogenous ligands promotes platelet hyperreactivity and thrombosis. Circ Res 2013; 112: 103-112.

Henn V, Steinbach S, Büchner K, et al. The inflammatory action of CD40 ligand (CD154) expressed on activated human platelets is temporally limited by coexpressed CD40. Blood 2001; 98: 1047-1054.

Gerdes N, Seijkens T, Lievens D, et al. Platelet CD40 exacerbates atherosclerosis by transcellular activation of endothelial cells and leukocytes. Arterioscler Thromb Vasc Biol 2016; 36: 482-490.

Zhang B, Wu T, Chen M, et al. The CD40/CD40L system: a new therapeutic target for disease. Immunol Lett 2013; 153: 58-61.

Lübbers J, Rodríguez E, van Kooyk Y. Modulation of immune tolerance via siglec-sialic acid interactions. Front Immunol 2018; 9: 2807.

Clemetson KJ, Clemetson JM, Proudfoot AEl, et al. Functional expression of CCR1, CCR3, CCR4, and CXCR4 chemokine receptors on human platelets. Blood 2000; 96: 4046-4054.

Ali RA, Wuescher LM, Worth RG. Platelets: essential components of the immune system. Curr Trends Immunol 2016; 16: 65-78.

Li C, Li J, Li Y, et al. Crosstalk between platelets and the immune system: old systems with new discoveries. Adv Hematol 2012; 2012: 384685.

Ribeiro LS, Migliari Branco L, Franklin BS. Regulation of innate immune responses by platelets. Front Immunol 2019; 10: 1320.

Smyth SS, Mcever RP, Weyrich AS, et al. Platelet functions beyond hemostasis. J Thromb Haemost 2009; 7 : 1759-1766.

Mezger M, Nording H, Sauter R, et al. Platelets and immune responses during thromboinflammation. Front Immunol 2019; 10: 1731.

Lam FW, Burns AR, Smith CW, et al. Platelets enhance neutrophil transendothelial migration via P-selectin glycoprotein ligand-1. Am J Physiol Heart Circ Physiol 2011; 300: H468-H475.

Clark SR, Ma AC, Tavener SA, et al. Platelet TLR4 activates neutrophil extracellular traps to ensnare bacteria in septic blood. Nat Med 2007; 13: 463-469.

Vanichakarn P, Blair P, Wu C, et al. Neutrophil CD40 enhances platelet-mediated inflammation. Thromb Res 2008; 122: 346-358. 
Weyrich AS, Elstad MR, McEver RP, et al. Activated platelets signal chemokine synthesis by human monocytes. J Clin Invest 1996; 97: 1525-1534.

Woller G, Brandt E, Mittelstädt J, et al. Platelet factor 4/CXCL4-stimulated human monocytes induce apoptosis in endothelial cells by the release of oxygen radicals. $J$ Leukoc Biol 2008; 83: 936-945.

Linke B, Schreiber Y, Picard-Willems B, et al. Activated platelets induce an anti-inflammatory response of monocytes/macrophages through cross-regulation of $\mathrm{PGE}_{2}$ and cytokines. Mediators Inflamm 2017; 2017: 1463216.

Chatterjee M, von Ungern-Sternberg SNI, Seizer $\mathrm{P}$, et al. Platelet-derived CXCL12 regulates monocyte function, survival, differentiation into macrophages and foam cells through differential involvement of CXCR4-CXCR7. Cell Death Dis 2015; 6: e1989.

Pierre S, Linke B, Suo J, et al. GPVI and thromboxane receptor on platelets promote proinflammatory macrophage phenotypes during cutaneous inflammation. J Invest Dermatol 2017; 137: 686-695.

Carestia A, Mena HA, Olexen CM, et al. Platelets promote macrophage polarization toward pro-inflammatory phenotype and increase survival of septic mice. Cell Rep 2019; 28: 896-908.

Xiang B, Zhang G, Guo L, et al. Platelets protect from septic shock by inhibiting macrophage-dependent inflammation via the cyclooxygenase 1 signalling pathway. Nat Commun 2013; 4: 2657.

Kaneider NC, Kaser A, Tilg $\mathrm{H}$, et al. CD40 ligand-dependent maturation of human monocyte-derived dendritic cells by activated platelets. Int J Immunopathol Pharmacol 2003; 16: 225-231.

Czapiga M, Kirk AD, Lekstrom-Himes J. Platelets deliver costimulatory signals to antigen-presenting cells: a potential bridge between injury and immune activation. Exp Hematol 2004; 32: 135-139.

Hamzeh-Cognasse H, Cognasse F, Palle S, et al. Direct contact of platelets and their released products exert different effects on human dendritic cell maturation. BMC Immunol 2008; 9: 54.

Du Y, Liu X, Guo S-W. Platelets impair natural killer cell reactivity and function in endometriosis through multiple mechanisms. Hum Reprod 2017; 32: 794-810.

Kopp H-G, Placke T, Salih HR. Platelet-derived transforming growth factor- $\beta$ down-regulates NKG2D thereby inhibiting natural killer cell antitumor reactivity. Cancer Res 2009; 69: 7775-7783.

Sadallah S, Schmied L, Eken C, et al. Platelet-derived ectosomes reduce NK cell function. J Immunol 2016; 197: 1663-1671.

Raiden S, Schettini J, Salamone G, et al. Human platelets produce granulocyte-macrophage colony-stimulating factor and delay eosinophil apoptosis. Lab Invest 2003; 83: 589-598.

Pitchford SC, Momi S, Giannini S, et al. Platelet P-selectin is required for pulmonary eosinophil and lymphocyte recruitment in a murine model of allergic inflammation. Blood 2005; 105: 2074-2081.

Karhausen J, Choi HW, Maddipati KR, et al. Platelets trigger perivascular mast cell degranulation to cause inflammatory responses and tissue injury. Sci Adv 2020; 6: eaay6314.

Gerdes N, Zhu L, Ersoy M, et al. Platelets regulate $\mathrm{CD}^{+}{ }^{+}$T-cell differentiation via multiple chemokines in humans. Thromb Haemost 2011; 106: 353-362.

Chapman LM, Aggrey AA, Field DJ, et al. Platelets present antigen in the context of MHC class I. J Immunol 2012; 189: 916-923.

Elzey BD, Tian J, Jensen RJ, et al. Platelet-mediated modulation of adaptive immunity. A communication link between innate and adaptive immune compartments. Immunity 2003; 19: 9-19.

Sprague DL, Elzey BD, Crist SA, et al. Platelet-mediated modulation of adaptive immunity: unique delivery of CD154 signal by platelet-derived membrane vesicles. Blood 2008; 111: 5028-5036.

Lisman T. Platelet-neutrophil interactions as drivers of inflammatory and thrombotic disease. Cell Tissue Res 2018; 371: 567-576.

Li J, Kim K, Barazia A, et al. Platelet-neutrophil interactions under thromboinflammatory conditions. Cell Mol Life Sci 2015; 72: 2627-2643.

Gros A, Syvannarath V, Lamrani L, et al. Single platelets seal neutrophil-induced vascular breaches via GPVI during immune-complex-mediated inflammation in mice. Blood 2015; 126: 1017-1026.

Allen N, Barrett TJ, Guo Y, et al. Circulating monocyte-platelet aggregates are a robust marker of platelet activity in cardiovascular disease. Atherosclerosis 2019; 282: 11-18.

Michelson AD, Barnard MR, Krueger LA, et al. Circulating monocyte-platelet aggregates are a more sensitive marker of in vivo platelet activation than platelet surface P-selectin: studies in baboons, human coronary intervention, and human acute myocardial infarction. Circulation 2001; 104: 1533-1537.

Passacquale G, Vamadevan P, Pereira L, et al. Monocyte-platelet interaction induces a pro-inflammatory phenotype in circulating monocytes. PLoS One 2011; 6: e25595.

Rong M, Wang C, Wu Z, et al. Platelets induce a proinflammatory phenotype in monocytes via the CD147 pathway in rheumatoid arthritis. Arthritis Res Ther 2014; 16: 478.

De Meyer GRY, De Cleen DMM, Cooper S, et al. Platelet phagocytosis and processing of $\beta$-amyloid precursor protein as a mechanism of macrophage activation in atherosclerosis. Circ Res 2002; 90: 1197-1204.

Laffont B, Corduan A, Rousseau M, et al. Platelet microparticles reprogram macrophage gene expression and function. Thromb Haemost 2016; 115: 311-323. 
Ando Y, Oku T, Tsuji T. Platelets attenuate production of cytokines and nitric oxide by macrophages in response to bacterial endotoxin. Platelets 2016; 27: 344-350.

Langer HF, Daub K, Braun G, et al. Platelets recruit human dendritic cells via Mac-1/JAM-C interaction and modulate dendritic cell function in vitro. Arterioscler Thromb Vasc Biol 2007; 27: 1463-1470.

Verschoor A, Neuenhahn M, Navarini AA, et al. A platelet-mediated system for shuttling blood-borne bacteria to $\mathrm{CD} 8 \alpha^{+}$dendritic cells depends on glycoprotein GPIb and complement C3. Nat Immunol 2011; 12: 1194-1201.

Nieswandt B, Hafner M, Echtenacher B, et al. Lysis of tumor cells by natural killer cells in mice is impeded by platelets. Cancer Res 1999; 59: 1295-1300.

Marx C, Novotny J, Salbeck D, et al. Eosinophil-platelet interactions promote atherosclerosis and stabilize thrombosis with eosinophil extracellular traps. Blood 2019; 134: 1859-1872.

$\mathrm{Hu} \mathrm{H}$, Zhu L, Huang Z, et al. Platelets enhance lymphocyte adhesion and infiltration into arterial thrombus. Thromb Haemost 2010; 104: 1184-1192.

Sadallah S, Amicarella F, Eken C, et al. Ectosomes released by platelets induce differentiation of CD4 ${ }^{+} \mathrm{T}$ cells into T regulatory cells. Thromb Haemost 2014; 112: 1219-1229.

Hamzeh-Cognasse H, Damien P, Chabert A, et al. Platelets and infections - complex interactions with bacteria. Front Immunol 2015; 6: 82.

Seyoum M, Enawgaw B, Melku M. Human blood platelets and viruses: defense mechanism and role in the removal of viral pathogens. Thromb J 2018; 16: 16.

Hottz ED, Bozza FA, Bozza PT. Platelets in immune response to virus and immunopathology of viral infections. Front Med 2018; 5: 121.

Speth C, Rambach G, Lass-Flörl C. Platelet immunology in fungal infections. Thromb Haemost 2014; 112: 632-639.

Alonso AL, Cox D. Platelet interactions with viruses and parasites. Platelets 2015; 26: 317-323.

Miajlovic H, Zapotoczna M, Geoghegan JA, et al. Direct interaction of iron-regulated surface determinant IsdB of Staphylococcus aureus with the GPIIb/IIla receptor on platelets. Microbiology 2010; 156: 920-928.

Fitzgerald JR, Loughman A, Keane F, et al. Fibronectin-binding proteins of Staphylococcus aureus mediate activation of human platelets via fibrinogen and fibronectin bridges to integrin GPIIb/IIla and IgG binding to the FcyRlla receptor. Mol Microbiol 2006; 59: 212-230.

Assinger A, Kral JB, Yaiw KC, et al. Human cytomegalovirus-platelet interaction triggers toll-like receptor 2-dependent proinflammatory and proangiogenic responses. Arterioscler Thromb Vasc Biol 2014; 34: 801-809. Chaipan C, Soilleux EJ, Simpson P, et al. DC-SIGN and CLEC-2 mediate human immunodeficiency virus type 1 capture by platelets. J Virol 2006; 80: 8951-8960.

Kraemer BF, Campbell RA, Schwertz $\mathrm{H}$, et al. Novel anti-bacterial activities of $\beta$-defensin 1 in human platelets: suppression of pathogen growth and signaling of neutrophil extracellular trap formation. PLoS Pathog 2011; 7: e1002355.

Solomon Tsegaye T, Gnirß K, Rahe-Meyer N, et al. Platelet activation suppresses HIV-1 infection of T cells. Retrovirology 2013; 10: 48.

Auerbach DJ, Lin Y, Miao $\mathrm{H}$, et al. Identification of the platelet-derived chemokine CXCL4/PF-4 as a broad-spectrum HIV-1 inhibitor. Proc Natl Acad Sci USA 2012; 109: 9569-9574.

Kho S, Barber BE, Johar E, et al. Platelets kill circulating parasites of all major Plasmodium species in human malaria. Blood 2018; 132: 1332-1344.

McMorran BJ, Wieczorski L, Drysdale KE, et al. Platelet factor 4 and Duffy antigen required for platelet killing of Plasmodium falciparum. Science 2012; 338: 1348-1351.

Keane C, Tilley D, Cunningham A, et al. Invasive Streptococcus pneumoniae trigger platelet activation via Toll-like receptor 2. J Thromb Haemost 2010; 8: 2757-2765.

de Stoppelaar SF, Claushuis TAM, Schaap MCL, et al. Toll-like receptor signalling is not involved in platelet response to Streptococcus pneumoniae in vitro or in vivo. PLoS One 2016; 11: e0156977.

van den Boogaard FE, Schouten M, de Stoppelaar SF, et al. Thrombocytopenia impairs host defense during murine Streptococcus pneumoniae pneumonia. Crit Care Med 2015; 43: e75-e83.

de Stoppelaar SF, Van't Veer C, Roelofs JJTH, et al. Platelet and endothelial cell P-selectin are required for host defense against Klebsiella pneumoniae-induced pneumosepsis. J Thromb Haemost 2015; 13: 1128-1138.

de Stoppelaar SF, van 't Veer C, Claushuis TAM, et al. Thrombocytopenia impairs host defense in Gram-negative pneumonia-derived sepsis in mice. Blood 2014; 124: 3781-3790.

Amison RT, O'Shaughnessy BG, Arnold S, et al. Platelet depletion impairs host defense to pulmonary infection with Pseudomonas aeruginosa in mice. Am J Respir Cell Mol Biol 2018; 58: 331-340.

Fox KA, Kirwan DE, Whittington AM, et al. Platelets regulate pulmonary inflammation and tissue destruction in tuberculosis. Am J Respir Crit Care Med 2018; 198: 245-255.

Rondina MT, Brewster B, Grissom CK, et al. In vivo platelet activation in critically ill patients with primary 2009 influenza A(H1N1). Chest 2012; 141: 1490-1495.

Boilard E, Paré G, Rousseau M, et al. Influenza virus H1N1 activates platelets through FcyRIIA signaling and thrombin generation. Blood 2014; 123: 2854-2863. 
Koupenova M, Corkrey HA, Vitseva O, et al. The role of platelets in mediating a response to human influenza infection. Nat Commun 2019; 10: 1780.

100 Pulavendran S, Rudd JM, Maram P, et al. Combination therapy targeting platelet activation and virus replication protects mice against lethal influenza pneumonia. Am J Respir Cell Mol Biol 2019; 61: 689-701.

101 Manne BK, Denorme F, Middleton EA, et al. Platelet gene expression and function in patients with COVID-19. Blood 2020; 136: 1317-1329.

102 Althaus K, Marini I, Zlamal J, et al. Antibody-induced procoagulant platelets in severe COVID-19 infection. Blood 2021; 137: 1061-1071.

103 Mejias A, Dimo B, Suarez NM, et al. Whole blood gene expression profiles to assess pathogenesis and disease severity in infants with respiratory syncytial virus infection. PLoS Med 2013; 10: e1001549.

104 Kullaya VI, de Mast Q, van der Ven A, et al. Platelets modulate innate immune response against human respiratory syncytial virus in vitro. Viral Immunol 2017; 30: 576-581.

105 Rødland EK, Ueland T, Pedersen TM, et al. Activation of platelets by Aspergillus fumigatus and potential role of platelets in the immunopathogenesis of aspergillosis. Infect Immun 2010; 78: 1269-1275.

106 Perkhofer S, Kehrel BE, Dierich MP, et al. Human platelets attenuate Aspergillus species via granuledependent mechanisms. J Infect Dis 2008; 198: 1243-1246.

107 Christin L, Wysong DR, Meshulam T, et al. Human platelets damage Aspergillus fumigatus hyphae and may supplement killing by neutrophils. Infect Immun 1998; 66: 1181-1189.

108 Cheepsattayakorn A, Cheepsattayakorn R. Parasitic pneumonia and lung involvement. BioMed Res Int 2014; 2014: 874021.

109 Lambrecht BN, Hammad H. The immunology of asthma. Nat Immunol 2015; 16: 45-56.

110 Tan R, Liew MF, Lim HF, et al. Promises and challenges of biologics for severe asthma. Biochem Pharmacol 2020; 179: 114012.

111 Bakakos A, Loukides S, Usmani OS, et al. Biologics in severe asthma: the overlap endotype - opportunities and challenges. Expert Opin Biol Ther 2020; 20: 1427-1434.

112 Kowal K, Pampuch A, Kowal-Bielecka O, et al. Platelet activation in allergic asthma patients during allergen challenge with Dermatophagoides pteronyssinus. Clin Exp Allergy 2006; 36: 426-432.

113 Duarte D, Taveira-Gomes T, Sokhatska O, et al. Increased circulating platelet microparticles as a potential biomarker in asthma. Allergy 2013; 68: 1073-1075.

114 Lommatzsch M, Schloetcke K, Klotz J, et al. Brain-derived neurotrophic factor in platelets and airflow limitation in asthma. Am J Respir Crit Care Med 2005; 171: 115-120.

115 Averill FJ, Hubbard WC, Proud D, et al. Platelet activation in the lung after antigen challenge in a model of allergic asthma. Am Rev Respir Dis 1992; 145: 571-576.

116 Hasegawa S, Tashiro N, Matsubara T, et al. A comparison of FceRI-mediated RANTES release from human platelets between allergic patients and healthy individuals. Int Arch Allergy Immunol 2001; 125: Suppl. 1, 42-47.

117 Ulfman LH, Joosten DPH, van Aalst CW, et al. Platelets promote eosinophil adhesion of patients with asthma to endothelium under flow conditions. Am J Respir Cell Mol Biol 2003; 28: 512-519.

118 Kornerup KN, Page CP. The role of platelets in the pathophysiology of asthma. Platelets 2007; 18: 319-328.

119 Takeda T, Morita $\mathrm{H}$, Saito $\mathrm{H}$, et al. Recent advances in understanding the roles of blood platelets in the pathogenesis of allergic inflammation and bronchial asthma. Allergol Int 2018; 67: 326-333.

120 Gomez-Casado C, Villaseñor A, Rodriguez-Nogales A, et al. Understanding platelets in infectious and allergic lung diseases. Int J Mol Sci 2019; 20: 1730.

121 Idzko M, Pitchford S, Page C. Role of platelets in allergic airway inflammation. J Allergy Clin Immunol 2015; 135: 1416-1423.

$122 \mathrm{Xu} \mathrm{W}$, Cardenes N, Corey C, et al. Platelets from asthmatic individuals show less reliance on glycolysis. PLoS One 2015; 10: e0132007.

123 Obeso D, Mera-Berriatua L, Rodríguez-Coira J, et al. Multi-omics analysis points to altered platelet functions in severe food-associated respiratory allergy. Allergy 2018; 73: 2137-2149.

124 Mitsui C, Kajiwara K, Hayashi H, et al. Platelet activation markers overexpressed specifically in patients with aspirin-exacerbated respiratory disease. J Allergy Clin Immunol 2016; 137: 400-411.

125 Laidlaw TM, Kidder MS, Bhattacharyya N, et al. Cysteinyl leukotriene overproduction in aspirin-exacerbated respiratory disease is driven by platelet-adherent leukocytes. Blood 2012; 119: 3790-3798.

126 Pitchford SC, Momi S, Baglioni S, et al. Allergen induces the migration of platelets to lung tissue in allergic asthma. Am J Respir Crit Care Med 2008; 177: 604-612.

127 Shah SA, Kanabar V, Riffo-Vasquez Y, et al. Platelets independently recruit into asthmatic lungs and models of allergic inflammation via CCR3. Am J Respir Cell Mol Biol 2021; 64: 557-568.

128 Amison RT, Momi S, Morris A, et al. RhoA signaling through platelet P2Y1 receptor controls leukocyte recruitment in allergic mice. J Allergy Clin Immunol 2015; 135: 528-538.

129 Hayashizaki K, Kimura MY, Tokoyoda K, et al. Myosin light chains 9 and 12 are functional ligands for CD69 that regulate airway inflammation. Sci Immunol 2016; 1: eaaf9154. 
Tian J, Zhu T, Liu J, et al. Platelets promote allergic asthma through the expression of CD154. Cell Mol Immunol 2015; 12: 700-707.

Pitchford SC, Riffo-Vasquez Y, Sousa A, et al. Platelets are necessary for airway wall remodeling in a murine model of chronic allergic inflammation. Blood 2004; 103: 639-647.

Amison RT, Cleary SJ, Riffo-Vasquez Y, et al. Platelets play a central role in sensitization to allergen. Am J Respir Cell Mol Biol 2018; 59: 96-103.

Dürk T, Duerschmied D, Müller T, et al. Production of serotonin by tryptophan hydroxylase 1 and release vic platelets contribute to allergic airway inflammation. Am J Respir Crit Care Med 2013; 187: 476-485.

Takeda T, Unno H, Morita H, et al. Platelets constitutively express IL-33 protein and modulate eosinophilic airway inflammation. J Allergy Clin Immunol 2016; 138: 1395-1403.

Maclay JD, McAllister DA, Johnston S, et al. Increased platelet activation in patients with stable and acute exacerbation of COPD. Thorax 2011; 66: 769-774.

Tokés-Füzesi M, Ruzsics I, Rideg 0 , et al. Role of microparticles derived from monocytes, endothelial cells and platelets in the exacerbation of COPD. Int J Chron Obstruct Pulmon Dis 2018; 13: 3749-3757.

Harrison MT, Short P, Williamson PA, et al. Thrombocytosis is associated with increased short and long term mortality after exacerbation of chronic obstructive pulmonary disease: a role for antiplatelet therapy? Thorax 2014; 69: 609-615.

Liu Y, Liu H, Li C, et al. Proteome profiling of lung tissues in chronic obstructive pulmonary disease (COPD): platelet and macrophage dysfunction contribute to the pathogenesis of COPD. Int J Chron Obstruct Pulmon Dis 2020; 15: 973-980.

Białas AJ, Siewiera K, Watała C, et al. Mitochondrial functioning abnormalities observed in blood platelets of chronic smoke-exposed guinea pigs - a pilot study. Int J Chron Obstruct Pulmon Dis 2018; 13: 3707-3717.

Fahim A, Crooks MG, Morice AH, et al. Increased platelet binding to circulating monocytes in idiopathic pulmonary fibrosis. Lung 2014; 192: 277-284.

Crooks MG, Fahim A, Naseem KM, et al. Increased platelet reactivity in idiopathic pulmonary fibrosis is mediated by a plasma factor. PLoS One 2014; 9: e111347.

O'Dwyer DN, Norman KC, Xia M, et al. The peripheral blood proteome signature of idiopathic pulmonary fibrosis is distinct from normal and is associated with novel immunological processes. Sci Rep 2017; 7: 46560.

Zhan T, Wei T, Dong L, et al. Cangrelor alleviates bleomycin-induced pulmonary fibrosis by inhibiting platelet activation in mice. Mol Immunol 2020; 120: 83-92.

Remková A, Šimková I, Valkovičová T. Platelet abnormalities in chronic thromboembolic pulmonary hypertension. Int J Clin Exp Med 2015; 8: 9700-9707.

Yaoita N, Shirakawa R, Fukumoto $\mathrm{Y}$, et al. Platelets are highly activated in patients of chronic thromboembolic pulmonary hypertension. Arterioscler Thromb Vasc Biol 2014; 34: 2486-2494.

gawa A, Matsubara H. Increased levels of platelet-derived microparticles in pulmonary hypertension. Thromb Res 2020; 195: 120-124.

Yadav H, Kor DJ. Platelets in the pathogenesis of acute respiratory distress syndrome. Am J Physiol Lung Cell Mol Physiol 2015; 309: L915-L923.

Middleton EA, Rondina MT, Schwertz $\mathrm{H}$, et al. Amicus or adversary revisited: platelets in acute lung injury and acute respiratory distress syndrome. Am J Respir Cell Mol Biol 2018; 59: 18-35.

Pitchford S, Cleary S, Arkless K, et al. Pharmacological strategies for targeting platelet activation in asthma. Curr Opin Pharmacol 2019; 46: 55-64.

Mallah H, Ball S, Sekhon J, et al. Platelets in chronic obstructive pulmonary disease: an update on pathophysiology and implications for antiplatelet therapy. Respir Med 2020; 171: 106098.

987-1002.

Koupenova M, Vitseva O, MacKay CR, et al. Platelet-TLR7 mediates host survival and platelet count during viral infection in the absence of platelet-dependent thrombosis. Blood 2014; 124: 791-802.

Aslan JE, McCarty OJ. Rho GTPases in platelet function. J Thromb Haemost 2013; 11: 35-46.

Moraes LA, Paul-Clark MJ, Rickman A, et al. Ligand-specific glucocorticoid receptor activation in human platelets. Blood 2005; 106: 4167-4175.

Liverani E, Banerjee S, Roberts W, et al. Prednisolone exerts exquisite inhibitory properties on platelet functions. Biochem Pharmacol 2012; 83: 1364-1373.

Visentin GP, Liu CY. Drug-induced thrombocytopenia. Hematol Oncol Clin North Am 2007; 21: 685-696.

Wang B, Peng Y, Dong J, et al. Human platelets express functional thymic stromal lymphopoietin receptors: a potential role in platelet activation in acute coronary syndrome. Cell Physiol Biochem 2013; 32: 1741-1750.

8 Fink L, Hölschermann H, Kwapiszewska G, et al. Characterization of platelet-specific mRNA by real-time PCR after laser-assisted microdissection. Thromb Haemost 2003; 90: 749-756.

Birschmann I, Mietner S, Dittrich M, et al. Use of functional highly purified human platelets for the identification of new proteins of the IPP signaling pathway. Thromb Res 2008; 122: 59-68. 